\title{
Penetrance of Methylene Tetrahydrofolate Reductase C677T Gene Polymorphism and Karyotypic Variations Associated Increase Genetic Susceptibility in the Cases of Congenital Heart Defects
}

\author{
Ajit K Saxena ${ }^{1 *}$, Sanjeev Kumar ${ }^{2}$, Meenakshi Tiwari ${ }^{1}$ and Chandan Kumar Singh ${ }^{1}$ \\ ${ }^{1}$ Department of Pathology /Lab Medicine, All India Institute of Medical Sciences, India \\ ${ }^{2}$ Department of Cardiothoracic and Vascular Surgery, All India Institute of Medical Sciences, India
}

*Corresponding author: Ajit Kumar Saxena, Professor and Head, Department of Pathology /Lab Medicine, All India Institute of

Medical Sciences, Patna - 801507 Bihar, India

\section{ARTICLE INFO}

Received: 幽 August 04, 2021

Published: 幽 August 13, 2021

Citation: Ajit K Saxena, Sanjeev Kumar, Meenakshi Tiwari, Chandan Kumar Singh. Penetrance of Methylene Tetrahydrofolate Reductase C677T Gene Polymorphism and Karyotypic Variations Associated Increase Genetic Susceptibility in the Cases of Congenital Heart Defects. Biomed J Sci \& Tech Res 38(1)-2021. BJSTR. MS.ID.006103.

Keywords: Congenital Heart Disease; Chromosomal Aberrations; FISH Analysis; Trisomy-21; MTHFR Gene Polymorphism

\begin{abstract}
Congenital heart disease (CHD), a multifaceted disorder occurs during embryogenesis due to exposure of environmental mutagens (teratogens) exposed antenatally leading to high-risk of infant mortality. Present study has been designed with the aims to evaluate the frequency of chromosome variation and corelate to methylene tetrahydrofolate reductase (MTHFR) C677T gene polymorphism as "risk factor" in clinically diagnosed cases of CHD using lymphocytes cultures and ARMS PCR, respectively. FISH analysis was carried for confirmation of chromosome-21. Interestingly, cytogenetics study shows variation in the frequency of structural and numerical chromosome aberrations with frequency in all the cases of CHD. Case-1, showing deletion of short arm of chromosome-18 and Robertsonian translocation between G/G chromosome association (24.00\%), Case-2 showing numerical variation (trisomy-21), Case-3, includes dicentric, chromatid break in chromosome-2, deletion of short arm in chromosome-5, reciprocal translocation involving chromosome- 6 and 10 and reporting first time appearance of ring of Y-chromosome. Case-4 showing structural variations $(16.00 \%)$ including dicentric, chromatid breaks and trisomy of chromosome-21. The most common dominant frequency was observed in karyotype trisomy-21(58.30\%) in all the four cases of CHD as an end point for genetic bio maker and showing significant differences $(\mathrm{p}<0.001)$ using X2- test between total number of chromosomes and trisomy-21 MTHFR (C677T) gene polymorphism reveals (25.00\%) of genetic heterozygosity of CT alleles and $75.00 \%$ cases shows homozygosity of wild type (CC) alleles, suggesting the variations in the frequency either in karyotypes or MTHFR C677T alleles are due to unconstitutional penetrance of gene in the genome of CHD cases and increase genetic susceptibility to make the disease more complex.
\end{abstract}

\section{Introduction}

Congenital heart defects (CHDs), the most severe congenital anomalies associated with "Birth Defect" in infants and their prevalence of 4 to 50 per 1000 live births and is associated with high morbidity and mortality [1]. CHD shows multiple gene(s) involvement during organogenesis including the environmental factors [2-4]. Interestingly, approximately $30 \%$ of children of CHDs shows chromosomal abnormality [1,3]. Among, the most common cytogenetic abnormality is the origin of trisomy-21(Down syndrome) in CHD cases [5]. Besides, chromosomal anomalies, 
role of 5,10-methylenetetrahydrofolate reductase (MTHFR) gene polymorphism associated DNA methylation has also been observed earlier in CHD cases [6-11].

In human, MTHFR gene is thermolabile in nature and present on chromosome $1 \mathrm{p} 3$ 6.3. The polymorphic variation of C677T allele (rs1801133) , the missense (point) mutation is responsible for the reduction enzymatic activity (30\% - 70\%) followed by increase "risk factor" in the variety of disease in heterozygous condition other than CHDs, such as neural tube defects (NTD), mental retardation, miscarriage and cancers [12-17]. Only few studies have been demonstrated regarding variations of MTHFR allele in CHD cases in Indian population [18,19]. Therefore, the present study has been designed to evaluate the frequency of karyotypic variations in individual case and try to find out their correlation with penetrance of MTHFR C677T gene polymorphism as "risk factor" in CHDs cases to confirm the hypothesis that congenital anomalies are polygenic in nature.

\section{Material and Methods}

Blood samples $(1.0 \mathrm{ml})$ were obtained in sterile heparinised vials from all the cases of $\mathrm{CHD}(\mathrm{n}=4)$ and their respective controls of the same age group after clinical diagnosis from the OPD of Cardiothoracic and Vascular Surgery (CTVS) Department and referred to Human Molecular Cytogenetic Laboratory of the Department of Pathology / Lab Medicine to evaluate the causative genetic risk factors, after clearance form Institute Ethical Committee (IEC), All India Institute of Medical Sciences, Patna.

\section{Cytogenetics Study}

Lymphocytes cultures were set up in the media (RPMI 1640) supplemented with foetal calf serum (5\%), Phytohaemagglutinin-M and antibiotic (penicillin \& streptomycin) for $72 \mathrm{hrs}$ in $\mathrm{CO}_{2}$ incubator at $37^{\circ} \mathrm{C}$ under sterile conditions. Harvesting was performed after adding colchicine $(10 \mu \mathrm{l})$ in the cultures to arrest mitosis. Prewarmed KCL (0.056\%) solution was used as hypotonic, and cells were fixed in acetic acid and methanol (1:3) as detailed procedure is illustrated earlier by Saxena et al in 2020. Giemsa stain was used for staining and GTG banding was performed after using $(0.001 \%)$ trypsin in PBS buffer ( $\mathrm{pH}$ 7.4) and karyotyping was performed according to the ISCN (2016) [20] using applied spectral imaging software (Genesis, USA).

\section{Fluorescence in-situ Hybridization (FISH).}

FISH analysis was carried out for the study of D21S65 specific region for chromosome-21 in both interphase and metaphases plates after using probe LSI 21 spectrum orange having region 21q22.13-q22.2 of $220 \mathrm{~kb}$, obtained from Abbott-Vysis, Inc. (USA). Chromosome - 21 was identified by green signal and nuclear DNA was labelled with DAPI as counter stain (Blue).

\section{SNP Analysis of MTHFR C677T Alleles}

MTHFR C677T gene polymorphism was carried out by RT RT-PCR (Bio Red, USA) using Syber green as fluorescence dye, procedure published earlier by our research group21. Genomic DNA were isolated using Promega DNA isolation Kit (USA) using manufacturer protocol and quantified using Nanodrop spectrophotometer (Thermo scientific, USA). The samples were stored at $-20^{\circ} \mathrm{C}$ till the PCR reaction was performed. MTHFR 677CT primers were designed for tetra plex RT PCR assay (http:// cedar.genetics.soton.ac.uk/public_html/primer1.html) and BLAST program at http://www.ncbi.nlm.nih.gov/blast to determine the specificity of the primers. To increase the specificity of the reaction a mismatch at the 2 positions of the 3 ' end both the allele-specific primers were selected and confirmed by software. The selection of the primers was based on the amplicons 'Tm' values and following primers used in present study:

\section{MTHFR-T,5'-GCACTTGAAGGAGAAGGTGTCTGCGGGCGT-3'.}

MTHFR-C-polyG,5'-GGCGGGCGGCCGGGAAAAGCTGCGTGATGAT GAAATAGG-3'.

\section{MTHFR-cf,5'-TGTCATCCCTATTGGCAGGTTACCCCAAA-3'.}

\section{MTHFR-cr,5'- CCATGTCGGTGCATGCCTTCACAAAG-3'.}

To obtain amplicons with distinct melting points, 'Tm' values were calculated using known software (http://eu.idtdna.com/ analyzer/Applications/OligoAnalyzer/). We have plan to select these primer ARMS PCR based on "Tm" analysis, part of plan of our interest to detect SNP of mutant of MTHFR allele(s), same PCR product was further confirmed by agarose gel $(2.0 \%)$ electrophoresis and bands were visualized and characterized after ethidium bromide staining on Gel Doc system.

Simple procedure includes a total volume of $20 \mu \mathrm{l}$ containing 10 $\mu \mathrm{l}$ of SYBR Green PCR Master Mix, $1 \mu$ of each primer per reaction, 40 ng of genomic DNA, and distilled water was taken to perform RT-PCR. The PCR protocol on the light cycler included an initial denaturation step (95 0C for $7 \mathrm{~min}$ ) was followed by amplification and quantification steps repeated for $30-40$ cycles $\left(95^{\circ} \mathrm{C}\right.$ for $10 \mathrm{~s}$, $60^{\circ} \mathrm{C}$ for $10 \mathrm{~s}, 72^{\circ} \mathrm{C}$ for $20 \mathrm{~s}$ ), with a single fluorescence measurement at the end of the elongation step at $72^{\circ} \mathrm{C}$, reaction was terminated by cooling to $40^{\circ} \mathrm{C}$. Melting curves were constructed by lowering the temperature to $65^{\circ} \mathrm{C}$ and later increasing the temperature by $0.2^{\circ} \mathrm{C} / \mathrm{s}$ to $98^{\circ} \mathrm{C}$ to measuring the change in fluorescence consistently. Tm values were assigned to develop plot generated by the RT-PCR of the negative derivation of fluorescence versus temperature $(\mathrm{dF} /$ dT) of the melting curve for amplification products measured at $530 \mathrm{~nm}$. 


\section{Statistical Analysis}

Chi square (X2) test was used to find to calculate $p$ value and level of significance ( $p=$ value) between normal and abnormal cells during analysis of karyotypes in individual case of CHDs.

\section{Results}

Cytogenetics analysis in CHD cases were performed for developing karyotypes individual to find end point according to ISCN 2016. The cytogenetic findings include a spectrum of GTG karyotypes showing large number of structural and numerical variations in individual cases of CHDs with brief history. The detail findings of numerical as well as structural variations (\% frequency) in individual cases were documented in Table 1.

\section{Case-I}

Three months old male child, clinically diagnosed CHD based on findings of ECHO cardiogram with AV+VA concordance and RA/ RV mildly enlarged. The cytogenetic study showing dominantly of $47 \mathrm{XY}+21$, confirmed down syndrome with the frequency of $58.3 \%$. Other trisomy identified were $47 \mathrm{XY}+20$ karyotype (4.16\%), 47 $\mathrm{XY}+18$ (4.16\%), $47 \mathrm{XY}+22$ (4.16\%), $47 \mathrm{XY}+9$ (4.16\%), $47 \mathrm{XY}+8$ (4.16\%), 47XY+11 4.16\%); $45 \mathrm{XY}-11(4.16 \%)$.

Table 1: Structural and numerical variation of chromosomes and their (\%) frequency in individual cases of Congenital Heart Defects.

\begin{tabular}{|c|c|c|c|c|c|}
\hline \multirow{2}{*}{ S.No; } & \multirow{2}{*}{ Karyotypes } & \multicolumn{4}{|c|}{ Number of aberrations \& their (\%) frequency } \\
\cline { 3 - 6 } & & Case-I (n=24) & Case-II (n=8) & Case-III (n=13) & Case-IV (n=12) \\
\hline 1 & $47, \mathrm{XY} / \mathrm{XX},+21 /$ & $14(58.30 \%)$ & $4(50.00 \%)$ & $2(15.30 \%)$ & 0 \\
\hline 2 & $47, \mathrm{XY},+20$ & $1(4.16 \%)$ & 0 & 0 & 0 \\
\hline 3 & $47, \mathrm{XY},+18$ & $1(4.16 \%)$ & 0 & 0 & 0 \\
\hline 4 & $47, \mathrm{XY},+22$ & $1(4.16 \%)$ & 0 & 0 & 0 \\
\hline 5 & $47, \mathrm{XY},+8$ & $1(4.16 \%)$ & 0 & 0 & 0 \\
\hline 6 & $47, \mathrm{XY},+9$ & $1(4.16 \%)$ & 0 & 0 & 0 \\
\hline 7 & $47, \mathrm{XY},+12$ & 0 & 0 & 0 & $1(7.69 \%)$ \\
\hline 8 & $47, \mathrm{XY},+14$ & 0 & $1(12.5 \%)$ & 0 & 0 \\
\hline 9 & $47, \mathrm{XX},+11$ & $1(4.16 \%)$ & 0 & 0 & 0 \\
\hline 10 & $47, \mathrm{XY},-11$ & $1(4.16 \%)$ & 0 & 0 & 0 \\
\hline 11 & $45, \mathrm{XY},-22$ & 0 & 0 & 0 & 0 \\
\hline 12 & $45, \mathrm{XY},-17$ & 0 & $8.30 \%)$ & 0 \\
\hline
\end{tabular}

\section{Case-II}

The one and half month-old, female child presented with CHD with clinical features including abnormal echocardiogram showing VSD (having thickness of $0.5 \mathrm{~cm}$ ) with clinical features of Down's syndrome The frequency of numerical variations having trisomy $21(47 \mathrm{XX}+21)$ were $50 \%$ and $47 \mathrm{XX}+11$ was $12.5 \%$.

\section{Case-III}

This case includes male, age two year and one month have AV malformations with multiple venous malformations in chest and abdominal wall with significant fatty components and diffusely infiltrated muscles (angiography). Karyotypes shows 15\% of cells with trisomy - $21(47 \mathrm{XY}+21)$ while trisomy $-12(47 \mathrm{XY}+12)$ is $7.7 \%$. In this case the structural chromosomal abnormities are dominant and shows more than $30 \%$, such as chromatid breaks of chromosome - 2 (2p) and chromosome 10 (10q) and formation of ring of $\mathrm{Y}$ chromosome. Besides these structural abnormalities one metaphase showed centromere breakage ( $>60 \%$ ) in chromosomes.

\section{Case-IV}

(P-593), Clinically diagnose on the bases of echocardiogram showing Situs Solitus, AV/VA concordance, Chamber size normal, valves normal. Cytogenetics findings includes $47 \mathrm{XY}+18$ (25\%); $47 \mathrm{XY}+14(8.3 \%), 45 \mathrm{XY}-11$ (8.3 \%) and 45XY -17 (8.3\%). Structural aberrations showing more than $8 \%$ chromatid breaks involving chromosome $1(1 \mathrm{p} 23)$

The variations in the frequency of chromosomes due to different clinical conditions and severity of disease. Interestingly, numerical variations and their frequency are predominantly dominated in all the cases of CHD including in the karyotypes of trisomy-21, 18, and monosomy of chromosome -11, a representative karyotype showing trisomy-21 is shown (Figure 1A). Simultaneously, structural chromosomal aberrations including chromatid breaks in chromosome $-1(-1 q)$ and $2(-2 p)$, besides this formation of Y-ring chromosome and centromeric breakage were the striking feature in two cases of CHD with frequency $>8 \%$ to $30 \%$, respectively. The numerical chromosomal variations were observed in all the 
four cases with maximum frequency (58.30\%) were observed in karyotypes $47, \mathrm{XY}+$ trisomy 21 . The extra copy of chromosome-21 was further confirmed by FISH analysis using specific probe LSI 21 spectrum orange having region 21q22.13-q22.2 of $220 \mathrm{~kb}$ as shown in Figure 1B. Interestingly, only one case showing lack of trisomy 21 , but extra copy of chromosome -18, 14 (trisomy) was observe with monosomy of chromosome $-11,17$, respectively.

Single nucleotide polymorphism (SNP) of MTHFR gene regulate folate metabolism is important event during embryogenesis. Highly sensitive, allele refractive mutation specific (ARMS) PCR based technique is used for SNP analysis to determine "risk factor" of MTHFR C677T genotyping - wild-type (CC) allele and mutant (TT) allele in homozygous condition and heterozygous condition (CT). In all the cases of CHDs, the Tm value showing shift from $82.5^{\circ} \mathrm{C}$ to $83.0^{\circ} \mathrm{C}$, suggesting increase heterozygocity (CT) in $25 \%$ of cases due to allele $\mathrm{C}$ change into $\mathrm{T}(\mathrm{C} \rightarrow \mathrm{T})$ as mentioned in Figure 2A, suggesting increase "risk" of the disease, while rest of the three cases (75\%) showing only wild type of genotype CC (Figure 2A2C). The findings RT-PCR were further confirmed the appearance of additional band of $105 \mathrm{~kb}$ belong to mutant "T" allele (Case - 3) on agarose gel electrophoresis as documented in Figure 2C.

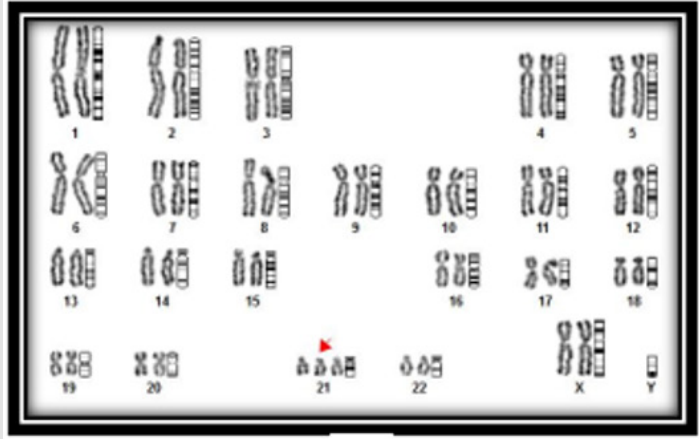

A

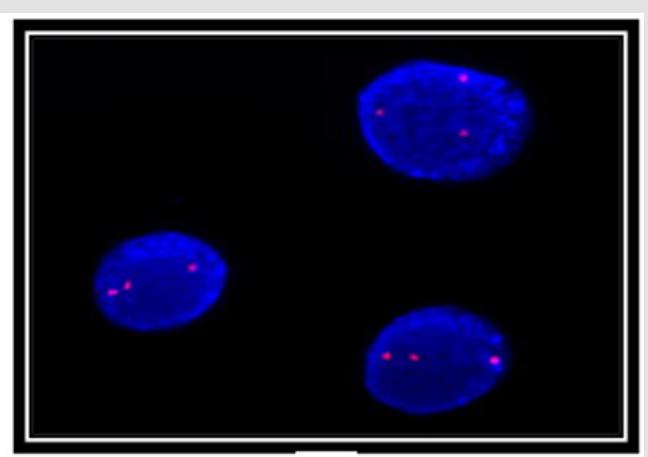

B

Figure 1: Karyotype $(46, X X+21)$ of Down syndrome (Figure.1A) and fluorescence in situ hybridization (FISH) analysis of interphase cell showing three positive signals (red) confirming extra of chromosome -21(Figure. B).

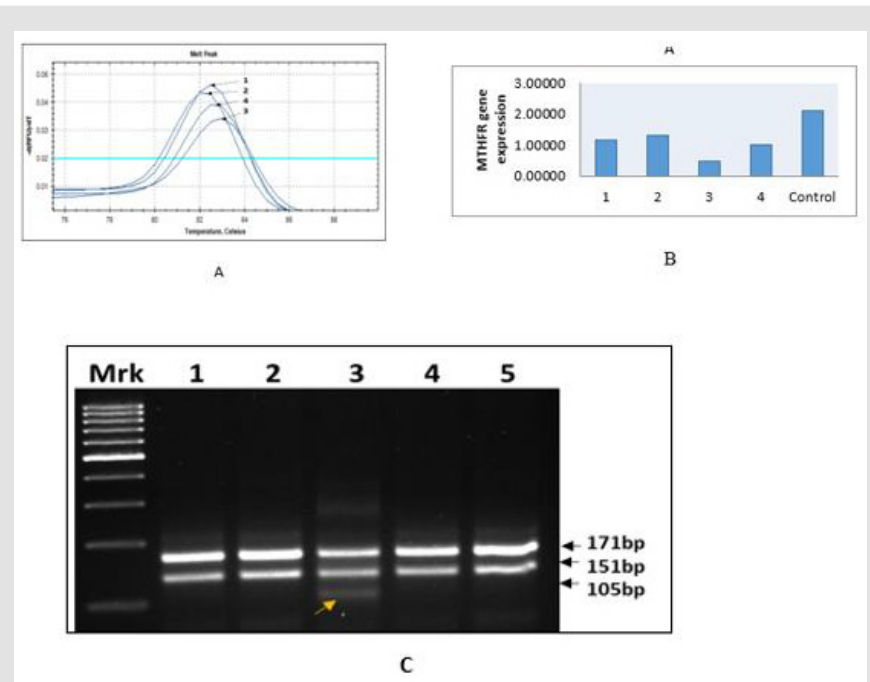

Figure 2: ARMS T-Plex real - time PCR assay for MTHFR C677T genotyping using wild-type (CC) and mutant (TT) allele, The effect of addition of a short GC tail on the Tm value of the $677 \mathrm{C}$ allele with melting-curve analysis for the allele specific and non-allele specific amplicons between cases and controls. Tm value showing shifting from 82.5 to 83.0 in the case -3 carrying the mutant allele " $\mathrm{T}$ " of heterozygous condition. Figure-2B. MTHFR gene expression using RT-PCR in different cases of congenital heart diseases (case 1-4) and compare with control. Case-3 showing significant down regulated the enzymatic activity due to heterozygous condition. (C) RT- PCR analysis of MTHFR C677T gene polymorphism are separated specific amplicons on agarose $(3 \%)$ gel, stained with ethidium bromide to visualized and characterized on Gel Doc system (Bio Rad USA). Lane-1,2,4, showing 677CC genotype (wild type) with $171 \mathrm{bp}$ and $150 \mathrm{bp}$, while, lane -3 carrying heterozygous $677 \mathrm{CT}$ allele (mutant type) with appearance additional band of 105bp in the case of congenital heart defects as compared to controls (lane-5) showing wild type (677CC) genotype. 


\section{Discussion}

The etiopathology of CHDs are complex due to the interactions of genetic and environmental factors [4,22]. It has been identified that $\sim 30 \%$ of CHD cases are syndromic involving chromosomal aberrations, while non-syndromic are monogenic and shows variety of genes. Now it is evident form preview of the fact that genetic factors become essential to understand the etiopathology in CHD cases and risk assessment before initiation of clinical management and counselling. However, there is lack of the genetic knowledge of CHDs in Indian population, only few studies have been achieved to study an association of genetic factors in CHDs. Therefore, present study was performed to accumulate the data from different approaches like karyotypic pattern and MTHFR (C677T) gene polymorphism to determine "risk factors" in CHD patients.

Epidemiological studies revel that frequency (\%) and types of chromosomal abnormalities varies between USA or Europe, but there is a lack of data in Indian population. Hence, the present study on CHDs becomes relevant to assess the risk using MTHFR C677T gene polymorphism analysis and try to correlate with karyotypic variations with agreement by the authors present study is small, but quite interesting. Previous studies identified chromosomal abnormalities with variation in the frequency from $3-18 \%$ in CHDs due to different variables such as clinical characteristics, age and ethnicity $[22,23]$. In the present study, numerical variations (trisomy-21) show very high frequency (58.30\%) and dominant over all kind of structural and numerical variations, the detail data of cytogenetics is documented in tabular form (table-I). Present findings are the agreement of earlier studies that trisomy-21 and 18 are associated with the cases of CHD [23,24]. Previous stud also reported trisomy-13, however, such chromosomal aberrations failed to observe in the present study may be either due to small sample size or different environmental factors [24].

In the present study, frequency of structural chromosomes was varying from $8-30 \%$ alone over total cytogenetics alterations and more interesting than numerical end point due to the appearance of "Y-ring chromosome and centromeric breakage reporting first time. Author failed to interpret the present findings that there might be involvement of conserve DNA sequences exist at centromeric regions and role of sex-chromosome during pathogenesis of CHD cases. Earlier study shows the deletion of the short arm of chromosome - 6 and 17, while present study also documented chromatid breaks - unstable regions of chromosomes [22-25]. Interestingly, present study shows "novel" structural changes has not been reported earlier with high impact on genomic instability of CHDs genome.

The conversion of folate 5,10-methylenetetrahydrofolate to 5-methyltetrahydrofolate in multistep processes that converts homocysteine to S-adenosyl methionine involving methyl donor for the maintenance of several biological processes. Methionine is incorporated into protein synthesis, growth and metabolism processes. The S-adenosyl methionine serves as a role for clearance of harmful substances, metabolites and waste products more efficiently. Earlier study shows that deficiency in MTHFR activity leads to increase homocysteine and lowering of folate levels in circulation. Homocysteine, is a Sulphur containing amino acid, acts as biomarker of "risk factor" of cardiovascular disease by promoting oxidation of lipids and lipoproteins [26]. Growing number of evidence also suggest that mutations in folate metabolism may also be involved in genetic susceptibility to CHD. In 2001, Junker and colleagues showed that significant association of MTHFR 677TT genotype in the development of CHDs during early pregnancy [8-11, 27]. There is still needed to explore new variants of MTHFR and their association to the "risk factor" during organogenesis in developing countries like India needs to be explored. In the present study, we report for the first time the role of MTHFR C677T polymorphism and codominance of chromosome variations in CHDs cases in the Eastern region of India. Previously, few studies have been reported mutant "T" allele of MTHFR in CHDs [18,19]. Our results indicate that the frequency of the putative risk allele C677T was 25\%, which is similar to meta-analysis study performed by Xuan et al where they identified frequency of $28.99 \%$ in Caucasian children and $31.76 \%$ in the Caucasian maternal population, although higher frequency (42.28\%) has been reported in Asian population [28]. Importantly, studies by Hobbs and co-workers identified Hcy, S-adenosylhomocysteine, and methionine as the most important biomarkers predictive in mothers whose pregnancies were affected by congenital heart defects (224 case subjects) or unaffected by any birth defect (90 control subjects)7. The variations in allele frequency may be either due to different clinical feature or penetrance of mutant allele to the CHDs genome to increase severity of the disease, but simultaneously the epigenetic factors should not be ignored.

\section{Conclusion}

From the present study we are reporting first time the penetrance of MTHFR C677T gene polymorphism as "risk factor" with karyotypic variations in the cases of CHDs. Although, this study is based on small group, but findings are promising with new findings in chromosome aberrations are documented as independent risk factor during embryogenesis, suggesting to increase large samples size for validity of the findings in other groups. However, other than genetic factor influences by consanguinity and environmental factors (socioeconomic) together cause genomic instability resulting abnormal development of foetus such as CHDs.

\section{Acknowledgement}

AKS thankfully acknowledges to the Director, AIIMS Patna also give special thanks to the patients who participates in the study. 


\section{Conflict of Interest}

All the authors have agreed with the content of the manuscript. There is no conflict of interest between the authors.

\section{Authors Contributions}

AKS and MT are involved for genetics analysis, $\mathrm{SK}$ is responsible for clinical diagnosis and management of the patients, while CK help during preparation of the manuscript.

\section{References}

1. Mary Ella Pierpont, Craig T Basson, D Woodrow Benson Jr, Bruce D Gelb, Therese M Giglia, et al. (2007) Genetic basis for congenital heart defects: current knowledge: a scientific statement from the American Heart Association Congenital Cardiac Defects Committee, Council on Cardiovascular Disease in the Young: endorsed by the American Academy of Pediatrics. Circulation 115(23): 3015-3038.

2. Richards AA, Garg V (2010) Genetics of congenital heart disease. Curr Cardiol Rev 6(2): 91-97.

3. Chaix MA, Andelfinger G, Khairy P (2016) Genetic testing in congenita heart disease: A clinical approach. World J Cardiol 8(2): 180-191.

4. Tian G, Gao H, Hu S, Shu Q (2018) Research progress on genetic and epigenetic mechanisms in congenital heart disease. Zhejiang Da Xue Xue Bao Yi Xue Ban 47(3): 227-238.

5. Benhaourech S, Drighil A, Hammiri AE (2016) congenital heart disease and Down syndrome: various aspects of a confirmed association. Cardiovasc J Afr 27(5): 287-290.

6. Zhu WL, Li Y, Yan L, Dao J, Li S (2006) Maternal and offspring MTHFR gene C677T polymorphism as predictors of congenital atrial septal defect and patent ductus arteriosus. Mol Hum Reprod 12(1): 51-54.

7. Charlotte A Hobbs, Mario A Cleves, Stepan Melnyk, Weizhi Zhao, S Jill James (2005) Congenital heart defects and abnormal maternal biomarkers of methionine and homocysteine metabolism. Am J Clin Nutr 81(1): 147-153.

8. Chrysovalanto Mamasoula, R Reid Prentice, Tomasz Pierscionek, Faith Pangilinan, James L Mills, et al. (2013) Association between C677T polymorphism of methylene tetrahydrofolate reductase and congenital heart disease: meta-analysis of 7697 cases and 13,125 controls. Circ Cardiovasc Genet 6(4): 347-353.

9. Wang W, Wang Y, Gong F, Zhu W, Fu S (2013) MTHFR C677T polymorphism and risk of congenital heart defects: evidence from 29 case-control and TDT studies. PLoS One 8(3): e58041.

10. Martí-Carvajal AJ, Solà I, Lathyris D, Dayer M (2017) Homocysteinelowering interventions for preventing cardiovascular events. Cochrane Database Syst Rev 8(8): CD006612.

11. Zhang R,Huo C, Wang X,Dang B, Mu Y, et al. (2018) Two Common MTHFR Gene Polymorphisms (C677T and A1298C) and Fetal Congenital Heart Disease Risk: An Updated Meta-Analysis with Trial Sequential Analysis. Cell Physiol Biochem 45(6): 2483-2496.

12. Saxena Ajit K (2011) MTHFR C677T gene mutation associated with severe risk for mental retardation in children. Int J of Genetics 3(2): 4749.

13. Gupta G, Gupta J, Pandey S, Pandey LK, Saxena Ajit K (2011) Role of Cell free fetal DNA in maternal blood - a prospective role of C $\beta S$ \& MTHFR gene as antenatal genetic marker in pre-eclampsia patients. Int. J. of Genet 3(2): 62-65.
14. Saxena Ajit K, Pandey S, Pandey LK (2012) Evaluation of Methylenetetrahydrofolate reductase C677T gene polymorphism associated risk factor in the patients of recurrent pregnancy loss. J. Med. Genet. Genomics 4(2): 25-28.

15. Singh A, Pandey S, Pandey LK, Saxena Ajit K (2015) In Human allele specific variation of MTHFR C677T and A1298C associate risk factor for the Development of Ovarian Cancer. J. Exp. Therap. Oncol 11(I): 67-70.

16. Saxena Ajit K, Tiwari M, Kumar V, Singh CK (2020) MTHFR New Gene Variants Increase Risk Factor in Wilms' tumor and Prediction of 3D Structure Modulates Functional Activity During Drug-Protein Interaction. J of Integrative Oncol 10: 1.

17. Saxena Ajit K, Agarwal M, Kumar A, Singh CK (2021) Genetic heterogenecity of MTHFR C677T allele modulate hormonal dysfunction associated risk factors in the cases of male infertility. Int J Dev Res 12 : $1-6$

18. Mukherjee M, Joshi S, Bagadi S, Dalvi M, Rao A, et al. (2002) A low prevalence of the C677T mutation in the methylenetetrahydrofolate reductase gene in Asian Indians. Clin Genet 61(2): 155-159.

19. Vasisht S, Gulati R, Narang R, N Srivastava,Lalit Mohan Srivastava, et al. (2002) Polymorphism (C677T) in the 5,10-methylenetetrahydrofolate reductase (MTHFR) gene: A preliminary study on north Indian men. Indian J Clin Biochem 17(1): 99-107.

20. Shaffer, Lisa G, Schmid M, McGowan-Jordan (2016) Iscn: An International System for Human Cytogenomic Nomenclature (2016). Basel: Karger.

21. Saxena Ajit K, Gupta RK, Kumar M (2016) ARMS-PCR based SNP analysis of MTHFR C677T allele using Syber green in pancreatic tumor. British Journal of Medicine and Medical Research 11(12): 1-6.

22. Robert J Hartman, Sonja A Rasmussen, Lorenzo D Botto, Tiffany Riehle-Colarusso, Christa L Martin, et al. (2011) The contribution of chromosomal abnormalities to congenital heart defects: a populationbased study. Pediatr Cardiol 32(8): 1147-1157.

23. Patrícia Trevisan,1 Tatiana Diehl Zen, Rafael Fabiano Machado Rosa,Juliane Nascimento da Silva,Dayane Bohn Koshiyama, et al. (2013) Chromosomal abnormalities in patients with congenital heart disease. Arq Bras Cardiol 101(6): 495-501.

24. C Ferencz, C A Neill, J A Boughman, J D Rubin, J I Brenner, L W Perry (1989) Congenital cardiovascular malformations associated with chromosome abnormalities: an epidemiologic study. J Pediatr 114(1): 79-86.

25. Paskulin GA, Zen PR, Rosa RF, Manique RC, Cotter PD (2007) Report of a child with a complete de novo $17 \mathrm{p}$ duplication localized to the terminal region of the long arm of chromosome 17. Am J Med Genet A 143A (12): 1366-1370.

26. P Frosst, H J Blom, R Milos, P Goyette, C A Sheppard, et al. (1995) A candidate genetic risk factor for vascular disease: a common mutation in methylenetetrahydrofolate reductase. Nat Genet 10(1): 111-113.

27. R Junker, S Kotthoff, H Vielhaber, S Halimeh, A Kosch, et al. (2001) Infant methylenetetrahydrofolate reductase 677TT genotype is a risk factor for congenital heart disease. Cardiovasc Res 51(2): 251-254.

28. Chao Xuan, Hui Li, Jin-Xia Zhao, Hong-Wei Wang, Yi Wang, et al. (2014) Association between MTHFR polymorphisms and congenital heart disease: a meta-analysis based on 9,329 cases and 15,076 controls. Sci Rep 4: 7311. 


\section{ISSN: 2574-1241}

DOI: 10.26717/BJSTR.2021.38.006103

Ajit K Saxena. Biomed J Sci \& Tech Res

(c) (9) This work is licensed under Creative

Submission Link: https://biomedres.us/submit-manuscript.php

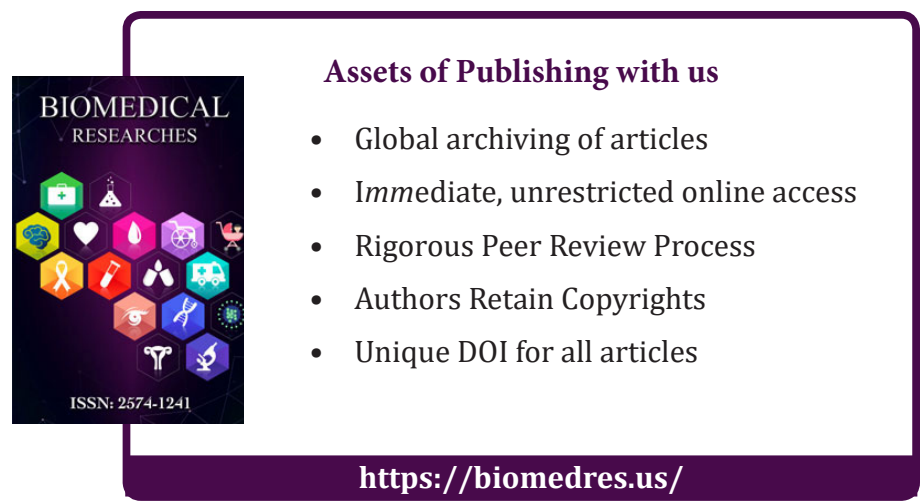

